\title{
Biofilms-producing Propionibacterium acnes attend at both inflamed and noninflamed acne lesions
}

Enea Gino Di Domenico ${ }^{1}$, Ilaria Cavallo ${ }^{1}$, Luigi Toma ${ }^{2}$, Grazia Prignano ${ }^{1}$, Maria Teresa Gallo $^{1}$, Fulvia Pimpinelli ${ }^{1}$, Valentina Bordignon ${ }^{1}$, Claudia Cavallotti ${ }^{3}$, Bruno Capitanio ${ }^{3}$, Fabrizio Ensoli ${ }^{1}$

1. Clinical Pathology and Microbiology, San Gallicano, Rome, Italy.

2. Infectious Disease Consultant, Regina Elena National Cancer Institute, Rome, Italy.

3. Department of Dermatology, San Gallicano, Rome, Italy.

\section{BACKGROUND}

Acne vulgaris is a chronic inflammatory disorder of the sebaceous follicles. Evidences suggest that Propionibacterium acnes plays an important role in the pathogenesis of acne vulgaris.

\section{OBJECTIVES}

To investigate the putative role of biofilm production by $P$. acnes in comedogenesis and in the progression towards inflamed acne lesions (Papule and Pustule).

\section{METHODS}

20 patient with different levels of acne. Samples were collected from healthy skin, microcomedon, comedon, papule and pustule in order to assess the presence of $P$. acnes.

\section{RESULTS}

\section{P. acnes can be isolated in $90 \%$ of patients}

Significant increase of $P$. acnes in the comedon and in the inflammatory lesions (Papule and Pustule)

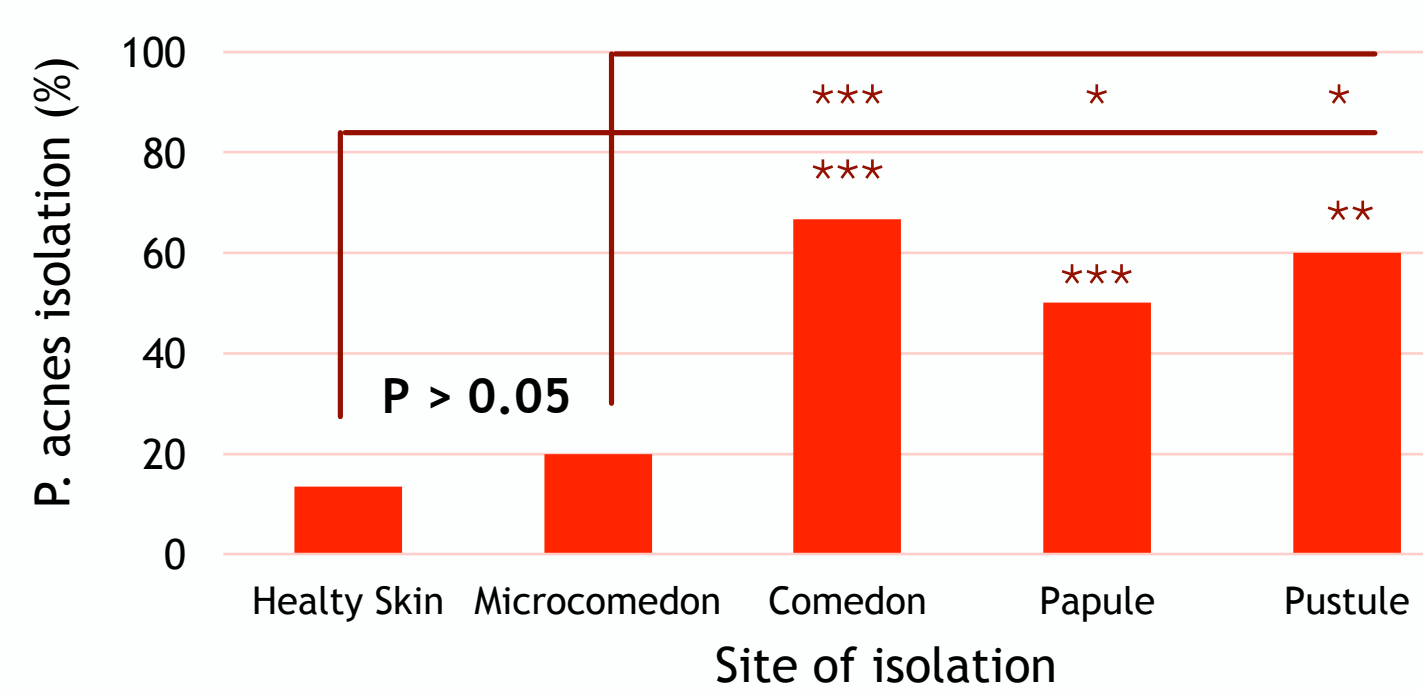

Prevalence of $P$. acnes is related to the single elementary lesion but not with the severity of acne

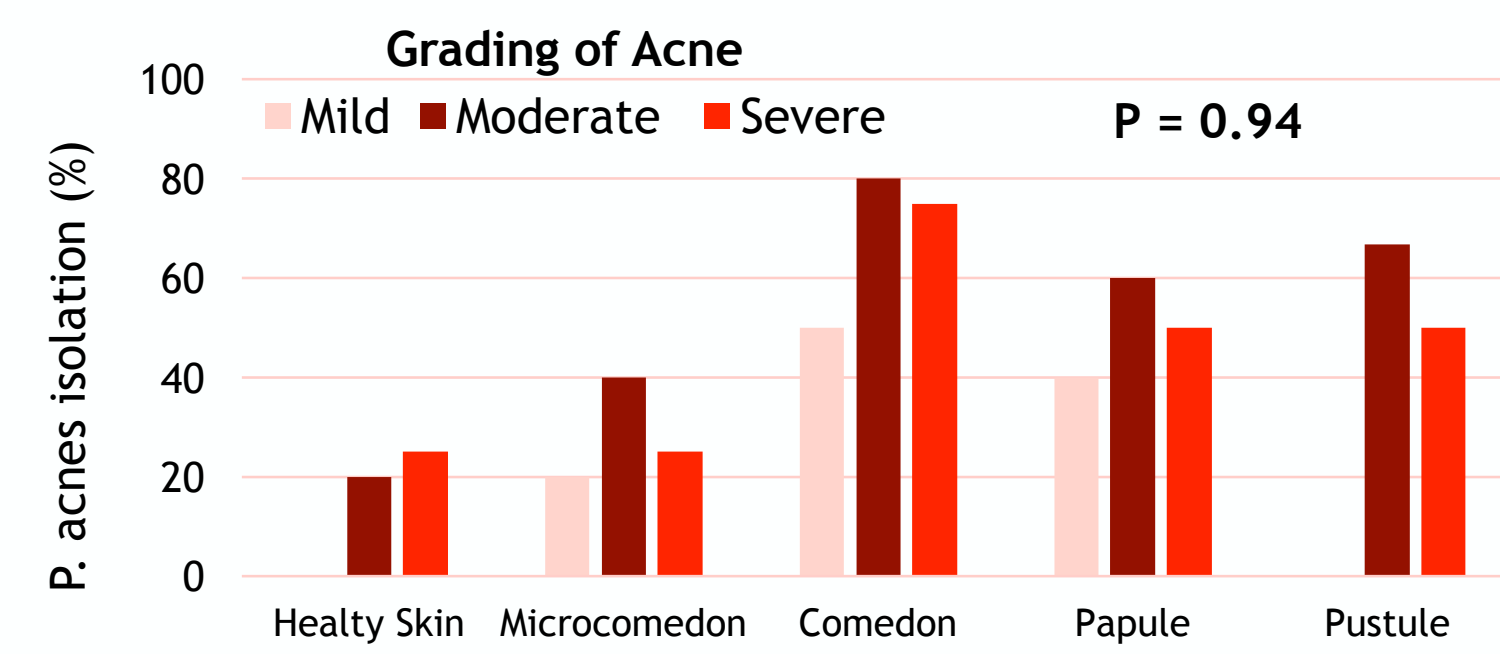

Site of isolation

All the $P$. acnes strains produce biofilm independently from the site of isolation

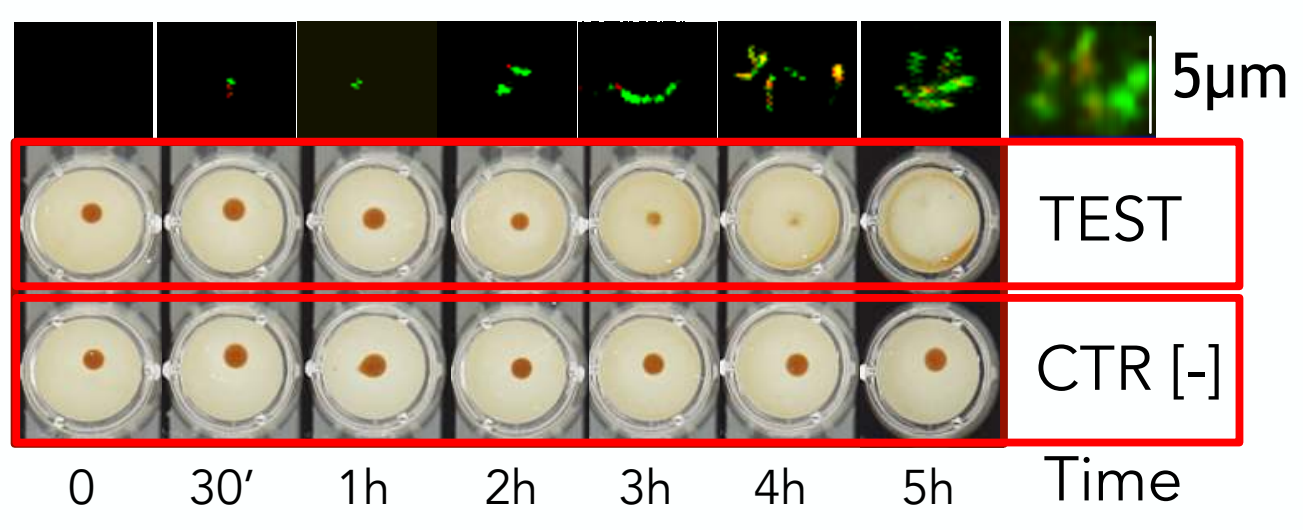

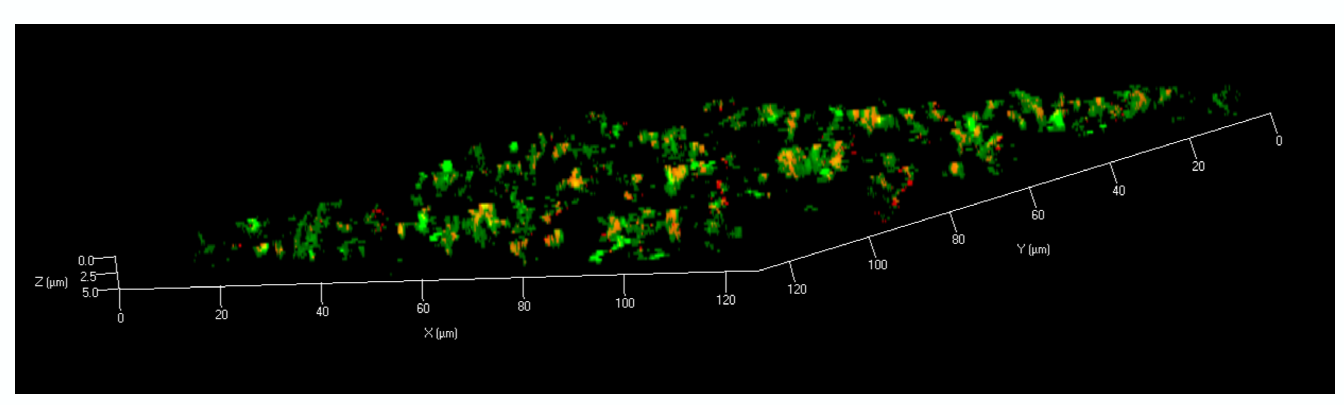

Mature biofilm after 24 hours

\section{CONCLUSIONS}

1) The prevalence of $P$. acnes depends on the type of the single elementary lesion

2) Biofilm plays a key role in promoting chronicity, supporting bacterial persistence 